
\title{
25 Research Square \\ Polymerase Acidic Subunit of H9N2 Polymerase Complex Induces Cell Apoptosis by Binding to PDCD 7 in A549 Cells
}

\section{Shaohua Wang}

Hebei North University https://orcid.org/0000-0002-4320-3532

$\mathrm{Na} \mathrm{Li}$

HEBEINU: Hebei North University

Shugang Jin

Hebei North University

Ruihua Zhang

HEBEINU: Hebei North University

Tong Xu ( $\nabla$ xutong1969@sohu.com )

Hebei North University

\section{Research}

Keywords: H9N2, Influenza A virus, PA subunit, programmed cell death protein 7, Cell apoptosis

Posted Date: November 18th, 2020

DOI: https://doi.org/10.21203/rs.3.rs-106350/v1

License: (c) (1) This work is licensed under a Creative Commons Attribution 4.0 International License. Read Full License

Version of Record: A version of this preprint was published at Virology Journal on April 13th, 2021. See the published version at https://doi.org/10.1186/s12985-021-01547-7. 


\section{Abstract}

Background: H9N2 influenza virus, a subtype of influenza A virus, can spread across different species and induce the respiratory infectious disease in humans, leading to a severe public health risk and a huge economic loss to poultry production. Increasing studies have shown that polymerase acidic (PA) subunit of RNA polymerase in ribonucleoproteins complex of H9N2 involves in crossing the host species barriers, the replication and airborne transmission of H9N2.

Methods: Here, to further investigate the role of PA subunit during the infection of H9N2 influenza virus, we employed mass spectrometry (MS) to search the potential binding proteins of PA subunit of H9N2. Our MS results showed that programmed cell death protein 7 (PDCD7) is a binding target of PA subunit. Co-immunoprecipitation and pull-down assays further confirmed the interaction between PDCD7 and PA subunit. Overexpression of PA subunit in A549 lung cells greatly increased the levels of PDCD7 in the nuclear and induced cell death assayed by MTT assay.

Results: Flow cytometry analysis and Western blot results showed that PA subunit overexpression significantly increased the expression of pro-apoptotic protein, bax and caspase 3 , and induced cell apoptosis. However, knockout of PDCD7 effectively attenuated the effects of PA overexpression in cell apoptosis.

Conclusions: In conclusion, the PA subunit of H9N2 bind with PDCD7 and regulated cell apoptosis, which provide new insights in the role of PA subunit during H9N2 influenza virus infection.

\section{Introduction}

Influenza is one of the most common infectious diseases, which mainly caused by the infection of influenza virus in seasonal epidemics [1,2]. There are four types of influenza viruses (A to $D)$, belonging to the RNA virus family Orthomyxoviridae, the genome of which contains eight segments of singlestranded negative-sense RNA [3, 4]. Influenza A virus mainly infects poultry, and some subtypes also infect mammals and humans. Different subtypes of influenza A virus come from different combinations of surface proteins, haemagglutinin (HA) and neuraminidase (NA) [3]. Among them, a subtype of influenza virus of influenza A, such as H9N2, induces avian influenza, which is a form of respiratory infectious disease $[5,6]$. The H9N2 subtype avian influenza virus has been reported to be low-pathogenic. Infection of H9N2 displays no clinical illness or only mild defects, such as a slight drop in egg production or mild respiratory signs, unless the infection is mixed with other pathogens, which will lead to severe respiratory syndromes and a high mortality rate [7]. Therefore, H9N2 avian influenza is a severe public health risk and will cause a huge economic loss to poultry production.

It is known that the genome of influenza A virus consists of eight ribonucleoproteins (RNPs), in which includes the genomic RNA, nucleoprotein, and an RNA polymerase [8]. The RNA polymerase in influenza A virus is a heterotrimer that composes of polymerase acidic (PA), polymerase basic 1 (PB1) and PB2. Accumulating studies have revealed that the polymerase complex is necessary for the viral host selection, 
replication, pathogenicity, and transmission $[9,10]$. Moreover, PA subunit of the RNA polymerase was reported to contribute to overcome the host species barrier and adapt to the new host species [11]. In addition, the polymorphisms in PA protein has also been identified to affect the pathogenicity and host adaptation of influenza A virus [12]. The PA subunit of the RNA polymerase is an endonuclease and a protease that induces proteolytic degradation of co-expressed proteins [13,14]. PA Subunit was reported to interact with a cellular protein with homology to a family of transcriptional activators [15]. However, other bind targets of PA subunit still need to be elucidated.

In the present study, mass spectrometry was employed to screen for the binding protein with PA subunit of viral RNA polymerase. We identified that programmed cell death protein 7 (PDCD7) is a new binding target of PA subunit of H9N2. Gain- and loss-of-function experiments demonstrated that PA subunit interacting with PDCD7 can activate apoptosis pathway and induce cell apoptosis, which may provide new insights in the role of PA subunit during H9N2 influenza virus infection.

\section{Material And Methods}

Cells and Cell culture.

A549 cells were purchased form American Type Culture Collection (ATCC® CCL-185 ${ }^{\mathrm{TM}}$ ). Cells were cultured with McCoy $[$ s 5A Media (Modified with Tricine) supplemented with $10 \%$ Fetal Bovine Serum (FBS) and $1 \%$ Penicillin-Streptomycin Solution. Cells were incubated at $37^{\circ} \mathrm{C}$ with $5 \%$ carbon dioxide and passaged by trypsinization.

Mass spectrometry.

For mass spectrometry analysis, lysates from A549 cells transfected with FLAG-tagged PA or vector control cells were prepared and incubated with FLAG-M2 agarose beads (Sigma) overnight at $4{ }^{\circ} \mathrm{C}$. The beads were then washed 3 times with IP wash buffer (150 mM NaCl, $10 \mathrm{mM} \mathrm{HEPES} \mathrm{pH} \mathrm{7.4,} \mathrm{0.1 \%} \mathrm{NP-40),}$ followed by elution with $1 \mathrm{M}$ glycine ( $\mathrm{pH}$ 3.0) twice. The eluted proteins were denatured and separated on SDS-polyacrylamide gels and stained with Coomassie blue; the indicated bands were subjected to mass spectrometry analysis.

\section{Co-immunoprecipitation}

Firstly, subunits of H9N2 polymerase were overexpressed in A549 cells and cultured for 48 hours, and then were collected via trypsinization. Total cell lysate was made with NP-40 lysis buffer and was quantitated by bicinchoninic acid (BCA). The overexpressed protein was precipitated by adding FLAG-M2 beads and incubating at $4^{\circ} \mathrm{C}$ overnight, then was isolated via centrifugation. The immunoprecipitation samples were analysed by SDS-PAGE and western blot.

\section{Pull-down assays}


Total protein lysate from A549 cells were as aforementioned. The purified GST-tagged protein was added into the cell lysate to bind interacted partners at $4^{\circ} \mathrm{C}$, GST beads were added to enrich the tagged complex, and the unspecific binding proteins were removed by multiple wash with washing buffer containing Tris- $\mathrm{HCl}$ and detergent. The samples were analysed by SDS-PAGE and western blot.

\section{MTT assay}

A549 cells were plated in 96-wells plated with complete medium with FBS and antibiotics, and the proliferation was analyzed by Cell Counting Kit -8 (CCK-8). Briefly, 20 ul CCK-8 reagent was added into each well and incubated at $37^{\circ} \mathrm{C}$ for 1 hours, absorbance at $450 \mathrm{nM}$ was then collected by the Molecular Device M2. Data was analyzed by GraphPad Prism 6.0.

\section{Flow cytometry analysis}

Cell apoptosis was analyzed by Annexin V and PI staining. Briefly, A549 cells were trypsinized and washed with PBS. Annexin V and PI reagent were added and incubated for 30 minutes form light. The fluorescence signals were analysed by BD Accuri ${ }^{\mathrm{TM}} \mathrm{C} 6$.

Quantitative real-time PCR.

The total RNA of A549 cells was extracted by Trizol method. Briefly: add $1 \mathrm{ml}$ Trizol to the cell culture dish to lyse and collect the cells, then add chloroform at the ratio of $200 \mathrm{ul} / 1 \mathrm{ml}$ Trizol, shake vigorously for 15 seconds, stand for 5 minutes and then centrifuge; take the upper aqueous phase into a new centrifuge tube and add equal volume of isopropanol to precipitate the RNA. The precipitate was collected by centrifugation after standing for 30 minutes at $4 \mathrm{C}$. The precipitate was washed with $75 \%$ ethanol without RNase and DNase and centrifuged again. The supernatant was discarded and dried and then dissolved in ultrapure water without RNase and DNase. cDNA was synthesized by reverse transcription form 2 UG of RNA, and $1 \mathrm{ul}$ of the synthesized cDNA was used as the template for real-time fluorescence quantitative PCR detection. PCR primers were as followed: GAPDH sense 5'- CAA GTT CAA CGG CAC AGT CA-3', GAPDH antisense 5'- ATC TCG CTC CTG GAA GAT GG-3'; ; PDCD7 sense primer: 5'- GGG ATC CAG ATG AGT TCC CA-3', PDCD7 antisense primer: 5'- ACG AAG TTG CCT TTG GGA TG-3'.

\section{Western blot}

Proteins samples were firstly separated with SDS-PAGE, and were transferred onto PVDF membranes. Sequentially, the membranes were blocked with $5 \%$ non-fat milk and then were incubated with Primary antibodies overnight at $4{ }^{\circ} \mathrm{C}$, Horseradish Peroxidase (HRP) conjugated secondary antibody was added and incubated for 1 hour at room temperature. Chemiluminescence signalling was collected via adding ECL reagents and the images was processed and analyzed with photoshop.

\section{CRISPR/Cas9 gene editing technology}

Guide RNA targeting the PDCD7 coding sequence was design on websites (Benchling.com) and was synthesized by micro-helix. PX459 vector containing both gRNA backbone and spCas 9 coding sequence was used to clone the designed gRNA and was confirmed via sanger sequencing. The gene editing 
plasmid was transfected into A549 cells using Lipo3000 and then were selected with puromycin at $1 \mathrm{ug} / \mathrm{ml}$ for 3 days. The survived cells were plated at low concertation allowing them into single clones after 2 weeks culture, and the knockout alleles were confirmed by Sanger sequencing and western blot.

\section{Statistical analysis}

All the data were expressed as mean \pm standard deviation. T test and a one-way ANOVA was used for the statistical analysis was performed by GraphPad 6.0.

\section{Results}

\section{PDCD7 is a binding target of PA subunit of H9N2.}

To investigate the role of the RNA polymerase in the RNP complex during the infection of H9N2 influenza virus, mass spectrometry (MS) was performed to search the binding protein of the RNA polymerase. We found that programmed cell death protein 7 (PDCD7) was pulled down by PA subunit antibody and identified by MS (Table 1, number), but other isoforms of PDCD were not observed, suggesting that PDCD7 may be a potential binding target of PA subunit.

Next, co-immunoprecipitation assay was performed to examine the interaction between PDCD7 and PA subunit. First, PDCD7 was co-transfected with PA-FLAG tag or empty vector into HEK293T cells. The immunoprecipitation was performed by using anti-FLAG antibody and then blotted with PDCD7 antibody. Interestingly, PDCD7 signal was observed in the PA-FLAG immunoprecipitates but not in vector control group (Fig. 1A), demonstrating an interaction between PDCD7 and PA subunit. To check whether this interaction is direct, we first purified PA with GST tag in E.coli cells and attempted to pull down His-tagged PDCD7 by using anti-His antibody. As shown in Fig. 1B, GST-PA antibody can pulldown PDCD7, confirming that PDCD7 is a direct binding target of PA subunit of H9N2.

\section{PA subunit of H9N2 greatly elevates the level of PDCD7 and induces cell death in lung cells.}

To examine whether overexpress PA subunits of H9N2 affects the expression level of PDCD7, the expression vector containing PA with Flag tag was transfected into A549 lung cells for $6 \mathrm{~h}, 12 \mathrm{~h}, 24 \mathrm{~h}$ and $48 \mathrm{~h}$. As shown in Fig. 2A, quantitative real-time PCR revealed that the expression of PDCD7 began to upregulate at $6 \mathrm{~h}$ and reached to a peak at $24 \mathrm{~h}$. Western blot results showed similar expression pattern of PDCD7 after PA overexpression (Fig. 2B), suggesting that overexpress PA subunits of H9N2 significantly upregulated PDCD7 expression in a time-dependent manner. Furthermore, immunohistochemistry results showed that PDCD7 signals (GFP) colocalized with PA signals (RFP) in the nuclear (Fig. 2E), although PA signals also existed in the plasma that may have other functions. These results indicated that PA subunits of H9N2 not only bind with PDCD7, but also elevated the expression level of PDCD7 in the nuclear.

Next, MTT assay was performed to determine the cell viability in A549 lung cells after overexpress PA subunits of H9N2. Consistent with the change of PDCD7 expression, the cell viability began to drop at 
$12 \mathrm{~h}$ and reached to a peak at $24 \mathrm{~h}$ (Fig. 2F), suggesting that PA overexpression induced cell death through binding and upregulating PDCD7.

\section{Overexpression of PA subunit induced cell apoptosis.}

PDCD7 is a protein that mediates cell apoptosis in response to various stimuli. To understand whether PA-overexpression-induced cell death resulted from cell apoptosis, annexin-PI staining combining with flow cytometry analysis was performed to determine the cell apoptosis. As shown in Fig. 3A and 3B, overexpression of PA subunit significantly induced cell apoptosis. Moreover, Western blot analysis demonstrated that the expression level of pro-apoptotic proteins, Bax and cleaved caspase-3, was significantly increased after PA overexpression, while the expression level of anti-apoptotic protein, Bcl-2, was decreased, compared to the vector control group (Fig. 3C), suggesting that overexpression of PA subunit activated apoptotic pathway and induced cell apoptosis.

\section{PDCD7 mediates the PA-overexpression-induced cell apoptosis in Lung A549 cells.}

To investigate whether PDCD7 mediated PA-overexpression-induced cell apoptosis in Lung A549 cells, CRISPR/Cas9 gene editing technology was used to selectively knock out PDCD7 expression. Western blot results showed that PDCD7 expression were successfully knocked out in Lung A549 cells (Fig. 4A). Next, PA-Flag tag vector was transfected into the PDCD7 knockout cell lines for $24 \mathrm{~h}$. Immunohistochemistry assay showed that PA signal was still observed in the nuclear, but do not bind with PDCD7 (Fig. 4B). Interestingly, PA-overexpression-induced cell apoptosis was great attenuated in PDCD7 knockout cell lines (Fig. 4B-C). Moreover, PA-overexpression-induced upregulation of BAX, cleaved caspase-3, and downregulation of Bcl-2 were also dramatically diminished (Fig. 4D-E). These results indicated that PDCD7 was downstream signal of PA subunit of H9N2 to activate cell apoptosis pathway and induce cell apoptosis.

\section{Discussion}

The avian H9N2 influenza virus belongs to the influenza A virus and was first isolated from the turkeys in Wisconsin in 1966 [16]. Since then, H9N2 virus becomes one of the most prevalent avian influenza viruses, causing repeated poultry and human infection [2, 7]. Although the H9N2 influenza virus has diversified into multiple lineages, H9N2 was reported to be low-pathogenic unless the viral infection is complicated with other pathogens [7, 17]. Avian H9N2 viruses can overcome the host species barrier and infect other mammals and humans[11, 18]. Accumulating studies have revealed that PA subunit of the RNA polymerase in influenza A virus are necessary in overcoming the host species barrier and host adaptation[11-15, 19,20], indicating that PA subunit is critical for the infection of avian H9N2 influenza virus. In the present study, combining MS with pull-down assay, we identified that PA subunit is capable of nuclear transport and binding with PDCD7 to activate apoptotic pathway in A549 lung cells.

The PA subunit of the RNA polymerase in influenza A virus is an endonuclease and a protease that induces proteolytic degradation of co-expressed proteins[13, 14]. It has been reported that amino acid 
residues in the N-terminus of the PA subunit is critical for the protein stability, endonuclease activity, cap binding, and protein binding [21]. Mutations in the PA subunit has been found to inhibit endonucleolytic cleavage of capped RNAs [22, 23]. Moreover, PA subunit of the RNA polymerase was also revealed to contribute to overcome the host species barrier and adapt to the new host species [11]. Mutations of PA subunit, such as PA-K356R, are necessary for viral adaptation to new hosts, and might render H9N2 viruses adapted for human infection [24]. In addition, the polymorphisms in PA protein also affects the pathogenicity and host adaptation of influenza A virus [12]. These evidences indicated that the PA subunit are critical for the function and infection of influenza A virus. A few binding proteins were identified to bind with PA subunit, such as a cellular protein with homology to a family of transcriptional activators [15]. PA subunit can also be translocated into the nuclei and regulate cRNA/vRNA synthesis [25]. Here, for the first time we demonstrated that PA subunit bind with PDCD7 in the nuclei and activated apoptosis pathway and induced cell apoptosis. The way how PA subunit bind with PDCD7 still needs to be further investigated.

PDCD7, also named as apoptosis-related protein ES 18, locates in chromosome 15 and encodes a $59 \mathrm{kDa}$ protein that interacts with the U11 small nuclear ribonucleoprotein, a component of the minor U12-type spliceosome, and contributes to the pre-mRNA splicing of U12-type introns [26-28]. Mouse PDCD7 was first identified using the screening approach in 1999 and characterized as an apoptosis-related protein that involves in apoptotic cell death of T-cells [29]. However, the specific function of PDCD7 in human is still unknown, although several studies have reported that human PDCD7 may related to the acute myeloid leukemia (AML) [30, 31]. Compared with the non-malignant samples, higher PDCD7 expression was found in AML patients and related to lower complete remission rate, shorter relapse-free survival, and overall survival, suggesting that overexpression of PDCD7 may serve as a molecular marker for prognosis estimation of AML $[30,31]$. By MS combining with Co-IP experiments, we found PDCD7 is a binding partner of PA subunit of avian H9N2 virus and mediates cell apoptosis after PA overexpression in A549 lung cells, indicating that the interaction between PDCD7 and PA subunit may contribute to the cell apoptosis during virus infection.

\section{Conclusion}

Collectively, the PA subunit of H9N2 might induce lung cell death through binding and activating PDCD7, which provide new insights in the role of PA subunit during H9N2 influenza virus infection and new treatment possibilities for H9N2 infected cases. Therefore, further study should be warranted.

\section{List Of Abbreviations}

PDCD7: programmed cell death protein 7

RNPs: ribonucleoproteins

PB1: polymerase basic 1 
ATCC: American Type Culture Collection

FBS: Fetal Bovine Serum

HRP: Horseradish Peroxidase

MS: mass spectrometry

AML: acute myeloid leukemia

\section{Declarations}

\section{Ethics approval and consent to participate}

Not applicable.

\section{Consent to publication}

Not applicable.

\section{Availability of data and material}

The data that support the findings of this study are available from the authors on request.

\section{Competing interests}

No conflict of interest exits in the submission of this manuscript, and manuscript is approved by all authors for publication.

\section{Acknowledgments}

Not applicable.

\section{Funding}

This work was supported by the National Natural Science Foundation of China [grant numbers 31672522, 31602030], Natural Science Foundation of Hebei Province, China [no. C2019405076, C2019405060], Special Funds for Layer and Broiler Innovation Team of the Second Phase of Agricultural Modernization Industry System of Hebei Province (Specialist Position for the Prevention and Control of Broiler Disease) [No.HBCT2018150207], The Natural Science Research Key Program of Educational Department of Hebei Province [ZD2019078]. The funder was not involved in the study design; the collection, analysis, and interpretation of data; in the writing of the report; and in the decision to submit the article for publication.

\section{Conflict of interest}

The authors have declared no conflict of interest. 
Authors' Contributions

Shaohua Wang and Na Li designed the project and conducted most of the laboratory work; Shugang Jin and Ruihua Zhang conducted the cell flow cytometry. Tong Xu contributed to data analysis and interpretation and writing the manuscript. All authors read and approved the final manuscript.

\section{References}

1. Moghadami M: A Narrative Review of Influenza: A Seasonal and Pandemic Disease.Iran J Med Sci 2017, 42:2-13.

2. Peacock THP, James J, Sealy JE, Iqbal M: A Global Perspective on H9N2 Avian Influenza Virus. Viruses 2019, 11.

3. Bouvier NM, Palese P: The biology of influenza viruses. Vaccine 2008, 26 Suppl 4:D49-53.

4. Szewczyk B, Bienkowska-Szewczyk K, Krol E: Introduction to molecular biology of influenza a viruses.Acta Biochim Pol 2014, 61:397-401.

5. Xia J, Cui JQ, He X, Liu YY, Yao KC, Cao SJ, Han XF, Huang Y: Genetic and antigenic evolution of H9N2 subtype avian influenza virus in domestic chickens in southwestern China, 2013-2016.PLOS One 2017, 12:e0171564.

6. Kandeil A, El-Shesheny R, Maatouq AM, Moatasim Y, Shehata MM, Bagato O, Rubrum A, Shanmuganatham K, Webby RJ, Ali MA, Kayali G: Genetic and antigenic evolution of H9N2 avian influenza viruses circulating in Egypt between 2011 and 2013.Arch Virol 2014, 159:2861-2876.

7. Naeem K, Ullah A, Manvell RJ, Alexander DJ: Avian influenza A subtype H9N2 in poultry in Pakistan. Vet Rec 1999, 145:560.

8. Ruigrok RW, Crepin T, Hart DJ, Cusack S: Towards an atomic resolution understanding of the influenza virus replication machinery.Curr Opin Struct Biol 2010, 20:104-113.

9. Gabriel G, Dauber B, Wolff T, Planz O, Klenk HD, Stech J: The viral polymerase mediates adaptation of an avian influenza virus to a mammalian host.Proc Natl Acad Sci U S A 2005, 102:18590-18595.

10. Steel J, Lowen AC, Mubareka S, Palese P: Transmission of influenza virus in a mammalian host is increased by PB2 amino acids 627K or 627E/701N.PLoS Pathog 2009, 5:e1000252.

11. Manz B, Schwemmle M, Brunotte L: Adaptation of avian influenza A virus polymerase in mammals to overcome the host species barrier.J Viro/ 2013, 87:7200-7209.

12. Naffakh N, Tomoiu A, Rameix-Welti MA, van der Werf S: Host restriction of avian influenza viruses at the level of the ribonucleoproteins.Annu Rev Microbio/ 2008, 62:403-424.

13. Dias A, Bouvier D, Crepin T, McCarthy AA, Hart DJ, Baudin F, Cusack S, Ruigrok RW: The capsnatching endonuclease of influenza virus polymerase resides in the PA subunit.Nature 2009, 458:914-918.

14. Perales B, Sanz-Ezquerro JJ, Gastaminza P, Ortega J, Santaren JF, Ortin J, Nieto A: The replication activity of influenza virus polymerase is linked to the capacity of the PA subunit to induce 
proteolysis.J Virol 2000, 74:1307-1312.

15. Huarte M, Sanz-Ezquerro JJ, Roncal F, Ortin J, Nieto A: PA subunit from influenza virus polymerase complex interacts with a cellular protein with homology to a family of transcriptional activators. $J$ Virol 2001, 75:8597-8604.

16. Homme PJ, Easterday BC: Avian influenza virus infections. I. Characteristics of influenza A-turkeyWisconsin-1966 virus.Avian Dis 1970, 14:66-74.

17. Jegede A, Fu Q, Berhane Y, Lin M, Kumar A, Guan J: H9N2 avian influenza virus retained low pathogenicity after serial passage in chickens. Can J Vet Res 2018, 82:131-138.

18. Schrauwen EJ, Fouchier RA: Host adaptation and transmission of influenza A viruses in mammals.Emerg Microbes Infect 2014, 3:e9.

19. Huarte M, Falcon A, Nakaya Y, Ortin J, Garcia-Sastre A, Nieto A: Threonine 157 of influenza virus PA polymerase subunit modulates RNA replication in infectious viruses.J Virol 2003, 77:6007-6013.

20. Maier HJ, Kashiwagi T, Hara K, Brownlee GG: Differential role of the influenza A virus polymerase PA subunit for VRNA and cRNA promoter binding. Virology 2008, 370:194-204.

21. Hara K, Schmidt FI, Crow M, Brownlee GG: Amino acid residues in the N-terminal region of the PA subunit of influenza A virus RNA polymerase play a critical role in protein stability, endonuclease activity, cap binding, and virion RNA promoter binding.J Virol 2006, 80:7789-7798.

22. Fodor E, Mingay LJ, Crow M, Deng T, Brownlee GG: A single amino acid mutation in the PA subunit of the influenza virus RNA polymerase promotes the generation of defective interfering RNAs.J Virol 2003, 77:5017-5020.

23. Fodor E, Crow M, Mingay LJ, Deng T, Sharps J, Fechter P, Brownlee GG: A single amino acid mutation in the PA subunit of the influenza virus RNA polymerase inhibits endonucleolytic cleavage of capped RNAs.J Virol 2002, 76:8989-9001.

24. Xu G, Zhang X, Gao W, Wang C, Wang J, Sun H, Sun Y, Guo L, Zhang R, Chang KC, et al: Prevailing PA Mutation K356R in Avian Influenza H9N2 Virus Increases Mammalian Replication and Pathogenicity.J Virol 2016, 90:8105-8114.

25. Nieto A, de la Luna S, Barcena J, Portela A, Ortin J: Complex structure of the nuclear translocation signal of influenza virus polymerase PA subunit.J Gen Virol 1994, 75 ( Pt 1):29-36.

26. Will CL, Schneider C, Hossbach M, Urlaub H, Rauhut R, Elbashir S, Tuschl T, Luhrmann R: The human 18S U11/U12 snRNP contains a set of novel proteins not found in the U2-dependent spliceosome. RNA 2004, 10:929-941.

27. Benecke $H$, Luhrmann R, Will CL: The U11/U12 snRNP 65K protein acts as a molecular bridge, binding the U12 snRNA and U11-59K protein.EMBO J 2005, 24:3057-3069.

28. Turunen JJ, Will CL, Grote M, Luhrmann R, Frilander MJ: The U11-48K protein contacts the 5 ' splice site of U12-type introns and the U11-59K protein.Mol Cell Biol 2008, 28:3548-3560.

29. Park EJ, Kim JH, Seong RH, Kim CG, Park SD, Hong SH: Characterization of a novel mouse CDNA, ES18, involved in apoptotic cell death of T-cells.Nucleic Acids Res 1999, 27:1524-1530. 
30. Tian Y, Huang Z, Wang Z, Yin C, Zhou L, Zhang L, Huang K, Zhou H, Jiang X, Li J, et al: Identification of novel molecular markers for prognosis estimation of acute myeloid leukemia: over-expression of PDCD7, FIS1 and Ang2 may indicate poor prognosis in pretreatment patients with acute myeloid leukemia.PLoS One 2014, 9:e84150.

31. Abo Elwafa R, Gamaleldin M, Ghallab O: The clinical and prognostic significance of FIS1, SPI1, PDCD7 and Ang2 expression levels in acute myeloid leukemia.Cancer Genet 2019, 233-234:84-95.

\section{Figures}

\section{Figure 1}

A

B
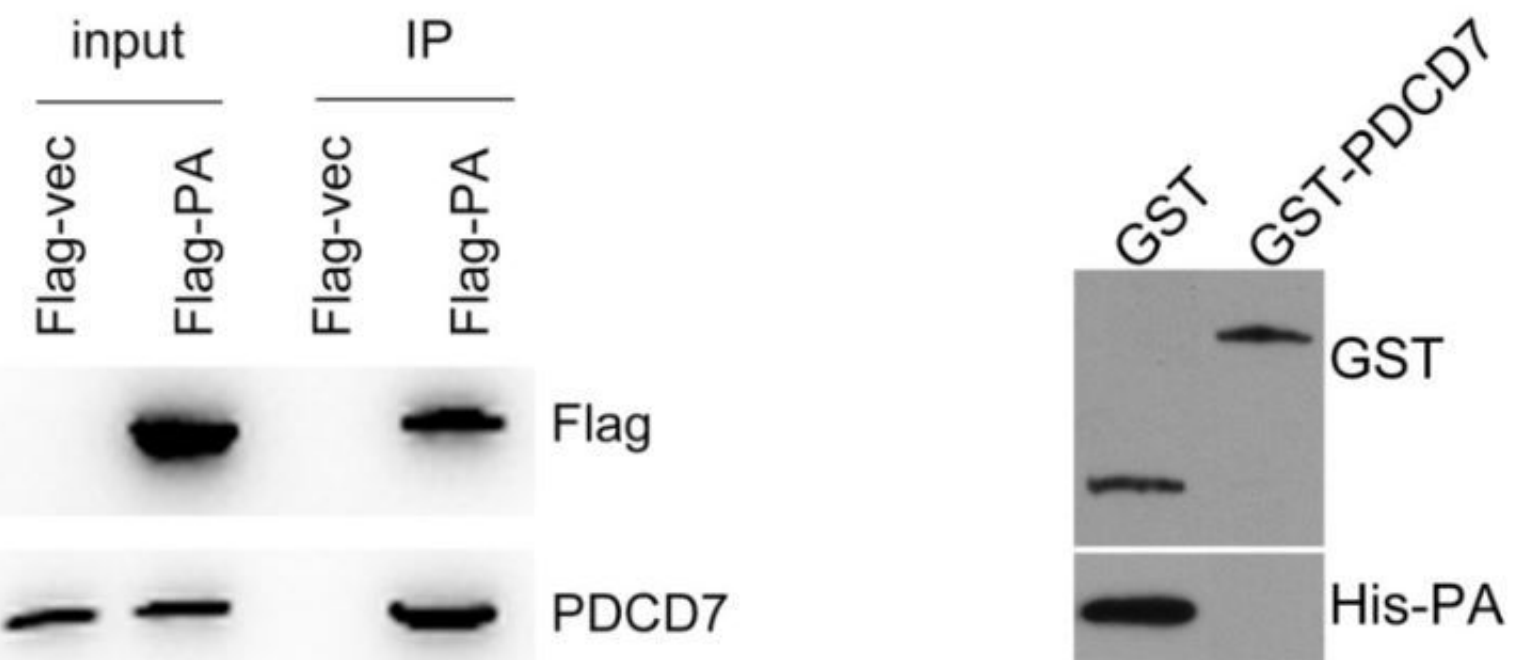

Figure 1

PDCD7 is a binding target of PA subunit of H9N2. A. Western blot detection of PDCD7 in the immunoprecipitation fraction of exogenous expressed Flag-PA. B. western blot detection of His-tagged PDCD7 in the fraction of GST-PA pull-down. 
Figure 2

A

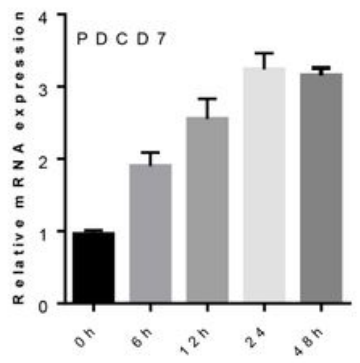

D

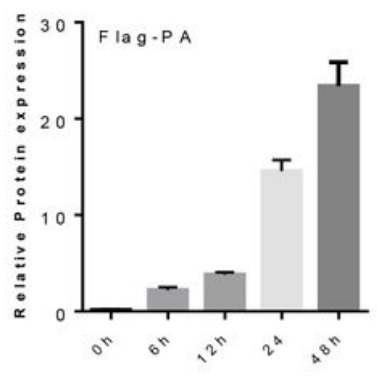

B

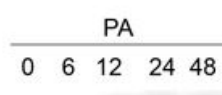

$-\infty \omega \mathrm{PDCD} 7$

$-\infty-\beta$-actin

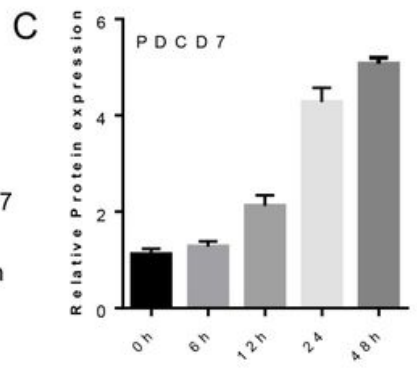

$\mathrm{E}$

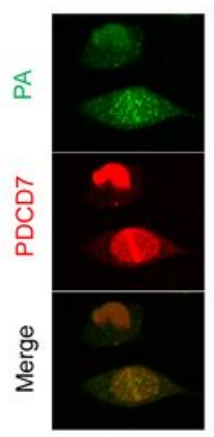

$\mathrm{F}$

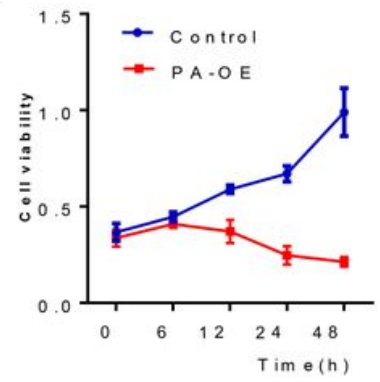

\section{Figure 2}

PA subunit of H9N2 greatly elevates the level of PDCD7 and induces cell death in lung cells. A.

Quantitative analysis of PDCD7 mRNA expression in lung cells upon PA overexpression. B-D. Western blot analysis of PDCD7 expression in A549 cells. E. colocalization of GFP-PA and RFP-PDCD7. F. Cell viability of A549 cells upon PA overexpression by MTT. 
Figure 3

A

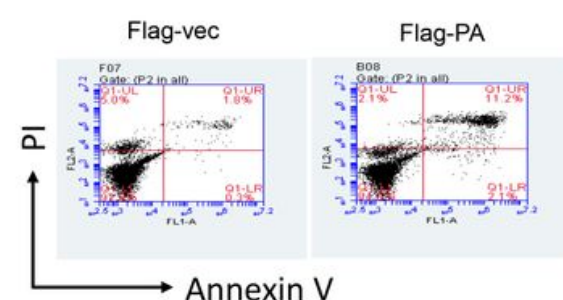

C

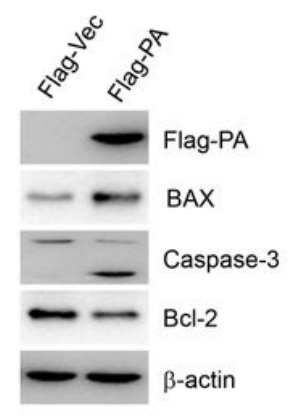

B

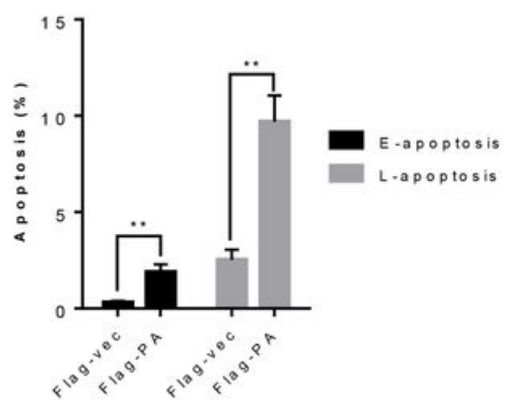

D

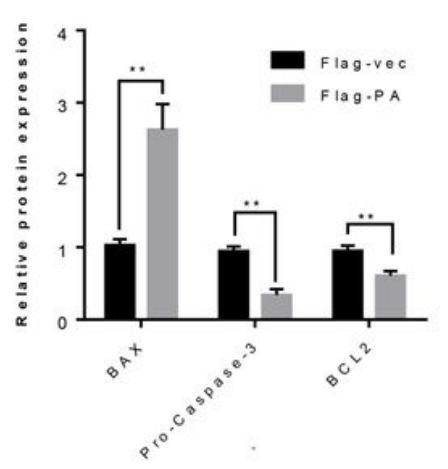

\section{Figure 3}

Overexpression of PA subunit induced cell apoptosis. A-B. Flow cytometry analysis of cell apoptosis by Annexin V and PI staining in A549 cells overexpressed with PA. C-D. Western blot analysis of apoptosis related proteins in A549 cells. “**” means $\mathrm{P}<0.01$. 
Figure 4

A

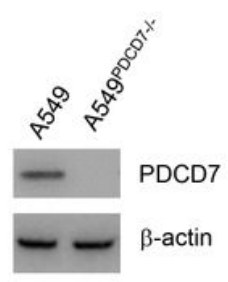

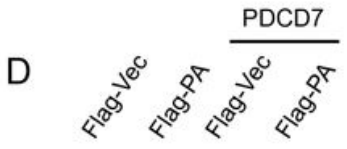

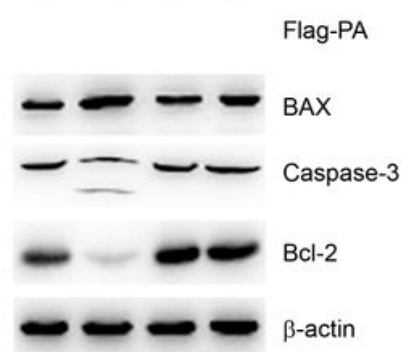

B

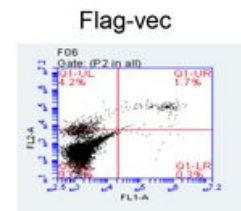

$\overline{0}$

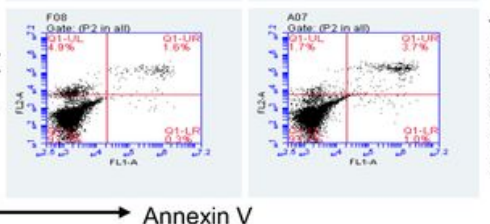

E

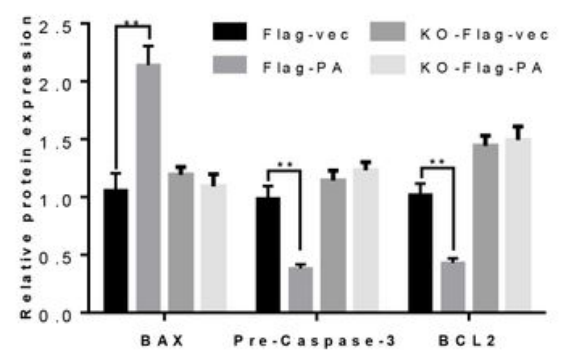

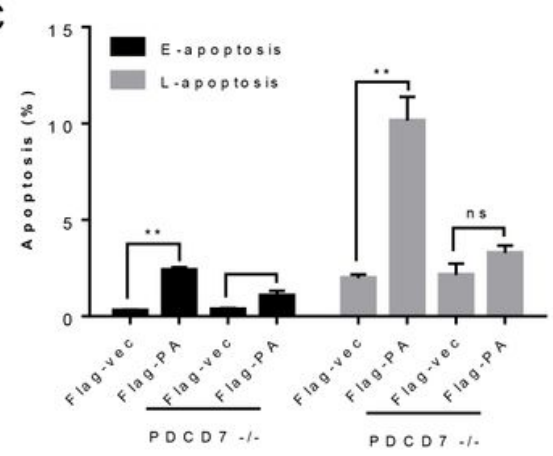

\section{Figure 4}

PDCD7 mediates the PA-overexpression-induced cell apoptosis in Lung A549 cells. A. Western blot analysis of PDCD7 knockout A549 cells. B-C. Flow cytometry analysis of A549 cells apoptosis. D-F. Western blot analysis of apoptosis related protein expression. 